Eur. J. Clin. Chem. Clin. Biochem.

Vol. 31, 1993, pp. 205-209

(C) 1993 Walter de Gruyter \& Co. Berlin - New York

\title{
Comparison of Urinary Neopterin and Pseudouridine in Patients with Malignant Proliferative Diseases*
}

\author{
By T. Motyl ${ }^{1}$, Zdzislawa Traczyk ${ }^{2}$,Wilhelmina Holska ${ }^{3}$, Dorota Daniewska-Michalska ${ }^{4}$, Stawomira Cieśluk ${ }^{2}$ \\ Wanda Kukulska ${ }^{1}$, Z. Kalużny ${ }^{2}$ and Malgorzata Podgurniak' \\ 1 Department of Animal Physiology, Veterinary Faculty, Warsaw Agricultural University, Warsaw, Poland \\ 2 IVth Division of Internal Diseases, Central Clinical Hospital, Warsaw, Poland \\ 3 Department 625, Tarchomin Pharmaceutical Works "Polfa", Warsaw, Poland \\ ${ }^{4}$ Department of Medicine, Warsaw Medical School, Central Hospital, Warsaw, Poland
}

(Received Seplember 18, 1992)**

Summary: The HPLC method for the simultaneous determination of urinary neopterin, pseudouridine, and creatinine allows a rapid evaluation of the activation state of cell-mediated immunity, and the stimulation of whole-body rRNA + tRNA turnover, associated with malignant growth.. Urinary neopterin and pseudouridine concentrations in healthy subjects amounted to: $106.6 \pm 34.6 \mu \mathrm{mol} / \mathrm{mol}$ creatinine, and $19.6 \pm 5.2 \mathrm{mmol} / \mathrm{mol}$ creatinine $(\overline{\mathrm{x}} \pm \mathrm{SD})$, respectively. The increase of neopterin excretion in patients with haematological neoplasms ranged from $146 \%$ in Hodgkin's disease to $534 \%$ in non-Hodgkin's lymphoma, whereas the increase in cancer cases ranged from $95 \%$ in adenocarcinoma of the gaster to $741 \%$ in hepatocellular carcinoma. The changes in pseudouridine excretion were much less pronounced: $63 \%$ in non-Hodgkin's lymphoma and $120 \%$ in carcinoma of the urinary bladder. The correlation coefficient between neopterin and pseudouridine was relatively low $(r=0.43)$, although statistically significant $(P<0.01)$. In the case of several neoplasms e.g. Hodgkin's disease, polycythaemia vera, and adenocarcinoma of the gaster, neopterin was significantly elevated, whereas pseudouridine remained at a normal concentration. There was a positive relationship between the stage of the disease (primary focus, regional metastases, dissemination) and urinary concentration of pseudouridine in patients with adenocarcinoma of the large intestine. In the same patients the increase of neopterin excretion was noticed both in early and advanced stages, with the highest values in disseminated disease.

\section{Introduction}

Neopterin and pseudouridine detected in biological fluids are used as biochemical markers of malignancy, but the source and mechanism of their elevation are different. Determination of neopterin in blood plasma and urine provides information about the activation state of cell-mediated immunity in vivo. Neopterin is synthesized from guanosine triphosphate by macrophages by the action of GTP cyclohydrolase, an enzyme induced by interferon gamma and derived from

* This study was supported by a grant from the Ministry of National Education.

** Submitted March 30/August 31, 1992 to "Pteridines" which discontinued with vol. 3, no. 3, 1992. activated T-lymphocytes (1). Thus, neopterin determined in blood serum (2) and urine (3) is considered as a good marker of $T$-cell activation. A significant diurnal rhythm of neopterin in urine has been detected with a peak at $06.30 \mathrm{~h}$ (4). Hausen et al. (5) has reviewed malignant diseases associated with increased urinary and/or serum neopterin concentrations: haematological neoplasms, gynaecological tumours, tumours of the genitourinary tract, lung cancer, gastrointestinal and pancratic carcinoma, melanomas, breast tumours, head and neck cancers.

The source of pseudouridine excreted in urine is whole-body catabolism of tRNA and rRNA (6). Pseudouridine is not re-utilized in nucleotide synthesis by 
the salvage pathway, appears in blood plasma, and is quantitatively excreted in urine (7). Pseudouridine is generally excreted in concentrations of 10 to 100 times those of other nucleosides, both in healthy subjects and cancer patients $(8,9)$. There is a considerable number of publications concerning changes in urinary pseudouridine excretion evoked by malignant growth $(9-15)$.

The present work was carried out to compare urinary neopterin and pseudouridine excretion in patients with haematological proliferative diseases and cancer. We offer a simple, sensitive and rapid HPLC method for the simultaneous determination of neopterin, pseudouridine and creatinine in the same urine sample.

\section{Materials and Methods}

\section{Patients}

Observations were performed on 130 individuals. The control group consisted of 31 healthy volunteers aged between 20 and 70 years.

The group with haematological neoplasms consisted of

52 patients:

15 with chronic lymphocytic leukaemia,

7 with multiple myeloma,

12 with other non-Hodgkin's lymphomas,

4 with myelofibrosis,

4 with Hodgkin's disease,

4 with chronic granulocytic leukaemia.

The group with cancer consisted of

47 patients:

7 with carcinoma of the lung,

10 of the rectum,

7 of the colon,

6 of the pancreas,

4 of the gaster,

3 of the liver (hepatocellular),

4 of the urinary bladder,

3 of the kidney,

3 of the testis.

\section{Urine collection and preparation}

The first morning urine of healthy individuals and patients with malignant proliferative diseases was stored at $-20^{\circ} \mathrm{C}$ in the dark prior to the analysis. Samples of urine were prepurified using Sep-Pak $C_{18}$ cartridges (Waters Assoc.). To protect neopterin from photooxidation, sample preparation was performed in dim light.

\section{Chromatographic method}

High performance liquid chromatography (HPLC) was performed with an isocratic liquid chromatograph Beckman 330 using an ultrasphere Altex column ODS $5 \mu \mathrm{m}, 4.6 \times 250 \mathrm{~mm}$, RP-18 cartridge precolumn $(4.6 \times 30 \mathrm{~mm})$, and RP-18 guard cartridge $(3.2 \times 15 \mathrm{~mm})$ - Pierce. The absorbance of pseudouridine and creatinine was measured by UV detector Beckman 153 equipped with a $254 \mathrm{~nm}$ filter. Effluent was then passed through a Shimadzu RF-535 fluorescence detector where neopterin was detected by its native fluorescence. Each detector was combined with an integrator-recorder Shimadzu C-R3A.

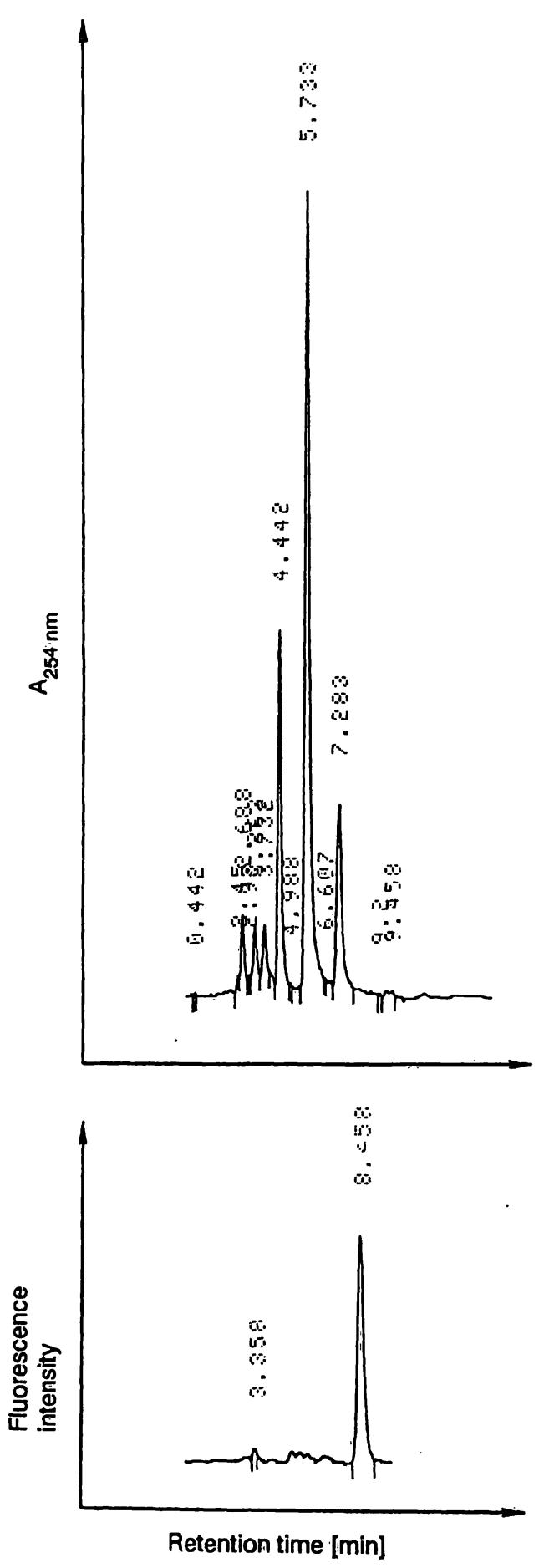

Fig. 1. HPLC chromatogram.

UV detection (upper): $3.73 \mathrm{~min}$ - orotic acid, $4.44 \mathrm{~min}$ - uric acid, $5.73 \mathrm{~min}$ - creatinine, $7.28 \mathrm{~min}$ - pseudouridine.

Fluorescence detection (lower): $8.46 \mathrm{~min}$ - neopterin. 
The mobile phase was $0.01 \mathrm{~mol} / \mathrm{l}$ potassium dihydrogen phosphate ( $\mathrm{pH}$ 5.9) containing methanol, volume fraction 0.01 , at a flow rate of $1 \mathrm{ml} \times \mathrm{min}^{-1}$. Samples were injected through an autosampler Spark-Holland 125 Fix equipped with dark glass vials and a $20 \mu \mathrm{lloop}$. An example of a urine HPLC chromatogram is presented in figure 1.

\section{Statistical analysis}

Results were statistically evaluated by ANOVA and Tukey range test (the significance of differences between the means of each group and subgroup of patients), and Student's t-test (the significance of differences between control group and each group of tumour patients). Correlation coefficients were calculated by the method of least squares. The probability of differences between averages at the level $P \leqslant 0.05$ was taken as significant, and at the level $P \leqslant 0.01$ as highly significant.

\section{Results and Discussion}

The HPLC method for the simultaneous determination of urinary neopterin, pseudouridine and creatinine (fig. 1) allows the rapid evaluation of the activation state of cell-mediated immunity and the rate of whole-body rRNA + tRNA turnover, associated with malignant growth. The values of urinary neopterin and pseudouridine concentrations in healthy subjects $(106.6 \pm 34.6 \mu \mathrm{mol} / \mathrm{mol}$ creatinine, and 19.5 $\pm 7.6 \mathrm{mmol} / \mathrm{mol}$ creatinine, respectively) are comparable to those described in earlier reports $(4,6)$.
Among haematological proliferative diseases, the highest increases $(P<0.01)$ of both analyses were observed in non-Hodgkin's lymphoma (neopterin: $675.7 \mu \mathrm{mol} / \mathrm{mol}$ creatinine; pseudouridine: $31.8 \mathrm{mmol} /$ mol creatinine), chronic lymphocytic leukaemia (neopterin: $438.4 \mu \mathrm{mol} / \mathrm{mol}$ creatinine, pseudouridine: $29.5 \mathrm{mmol} / \mathrm{mol}$ creatinine), myelofibrosis, and multiple myeloma (fig. 2).

The increase of urinary neopterin excretion was evident $(\mathrm{P}<0.01)$ in all examined cases of cancer (fig. 2) with the highest values in hepatocellular carcinoma $(897.0 \mu \mathrm{mol} / \mathrm{mol}$ creatinine), adenocarcinoma of the pancreas $(632.7 \mathrm{nml} / \mathrm{mmol}$ creatinine), adenocarcinoma of the rectum $(609.1 \mu \mathrm{mol} / \mathrm{mol}$ creatinine $)$, and adenocarcinoma of the colon $(566.8 \mu \mathrm{mol} / \mathrm{mol}$ creatinine). The highest concentrations of urinary pseudouridine were observed in carcinoma of the urinary bladder ( $42.8 \mathrm{mmol} / \mathrm{mol}$ creatinine), and hepatocellular carcinoma $(37.5 \mathrm{mmol} / \mathrm{mol}$ creatinine).

Generally, the changes of urinary neopterin and pseudouridine excretion showed a similar trend, but not in all examined patients. This reflected in a relatively low, although statistically significant, correlation coefficient $(r=0.43, P<0.01)$ between urinary neopterin and pseudouridine excretion. In several neo-

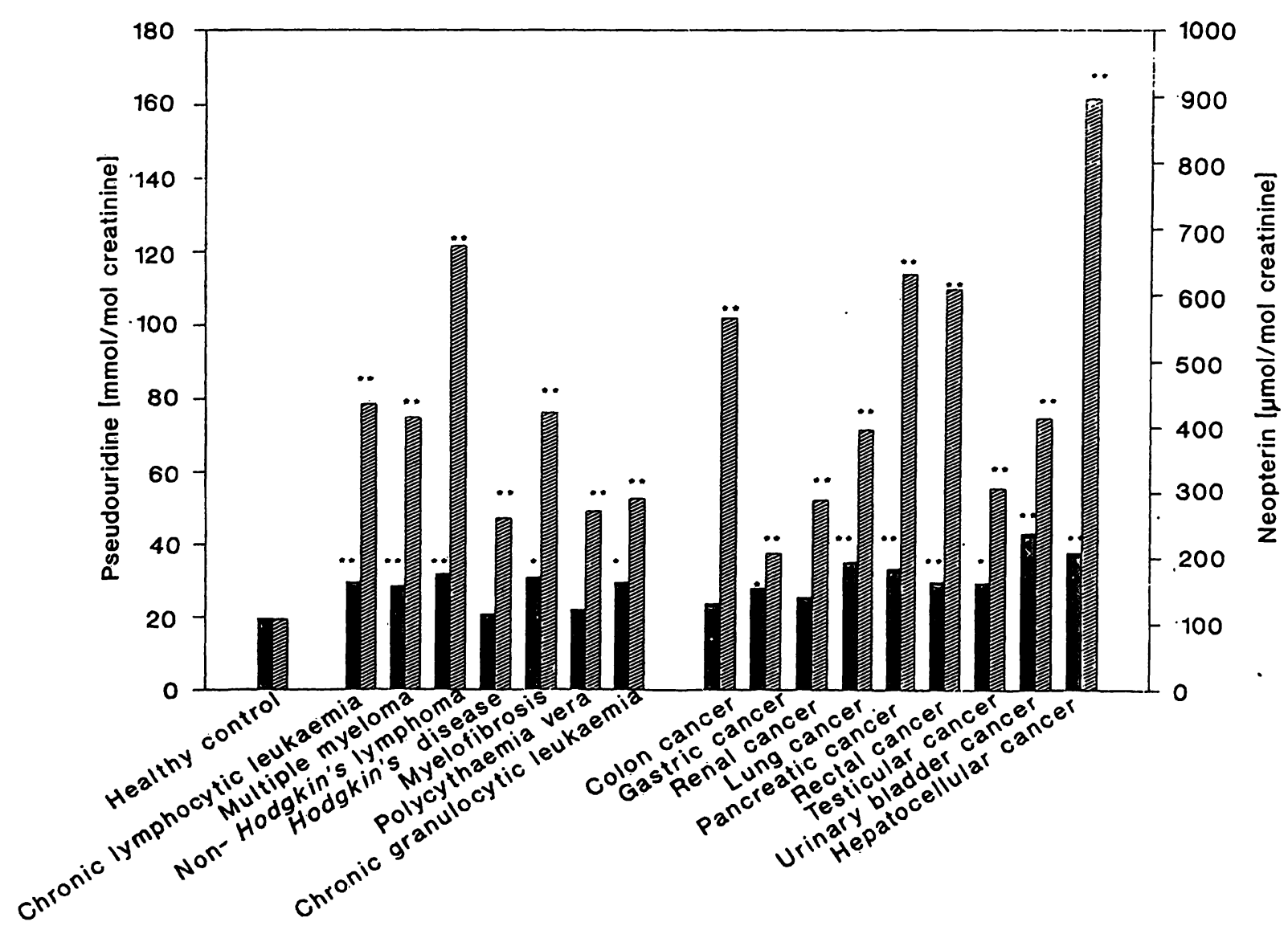

Fig. 2. The average urinary neopterin and pseudouridine concentration in patients with haematological proliferative diseases and cancers.

* values significantly $(P \leqslant 0.05)$ different from the control;

** values highly significantly $(P \leqslant 0.01)$ different from the control. 
plasms, e.g. Hodgkin's disease, polycythaemia vera, and adenocarcinoma of the gaster, neopterin was significantly elevated, whereas pseudouridine remained at a normal concentration. This confirms our findings of a poor response of blood plasma pseudouridine in the mentioned diseases (unpublished data). It should also be noted that elevations in urinary neopterin are much more pronounced than those of pseudouridine, both in haematological neoplasms and examined cancers (fig. 2). The increase (expressed as \% increase) of neopterin excretion in haematological proliferative diseases ranged from $146 \%$ in Hodgkin's disease to $534 \%$ in non-Hodgkin's lymphoma, whereas the highest increase of pseudouridine excretion in non-Hodgkin's lymphoma was $62 \%$. Similarly, in cancer patients, the increase of neopterin excretion ranged from $95 \%$ in adenocarcinoma of the gaster to $741 \%$ in hepatocellular carcinoma, but the maximal increase of pseudouridine excretion of $120 \%$ was observed in carcinoma of the urinary bladder. This is also illustrated by figure 3 , where urinary excretion of neopterin and pseudouridine is presented in relation to the stage of adenocarcinoma of the large intestine. If the particular stages described as primary focus, regional metastases, and dissemination are assigned hypothetical values of 1, 2 and 4, respectively, the relationship between the stage and urinary pseudouridine excretion is statistically significant $(r=0.60$, $P<0.01$ ). It reflects the progressive increase of $r R N A$ + tRNA turnover, associated with tumour burden. There was no such relationship between the stage of the disease and urinary neopterin, which was elevated both in early and advanced stages of malignant growth (fig. 3). It seems important that both markers provide to some extent independent information about malignant growth (pseudouridine) and immune activation (neopterin) in the same patient. The lack of correlation between the changes observed in pseudouridine and neopterin in subgroups of patients

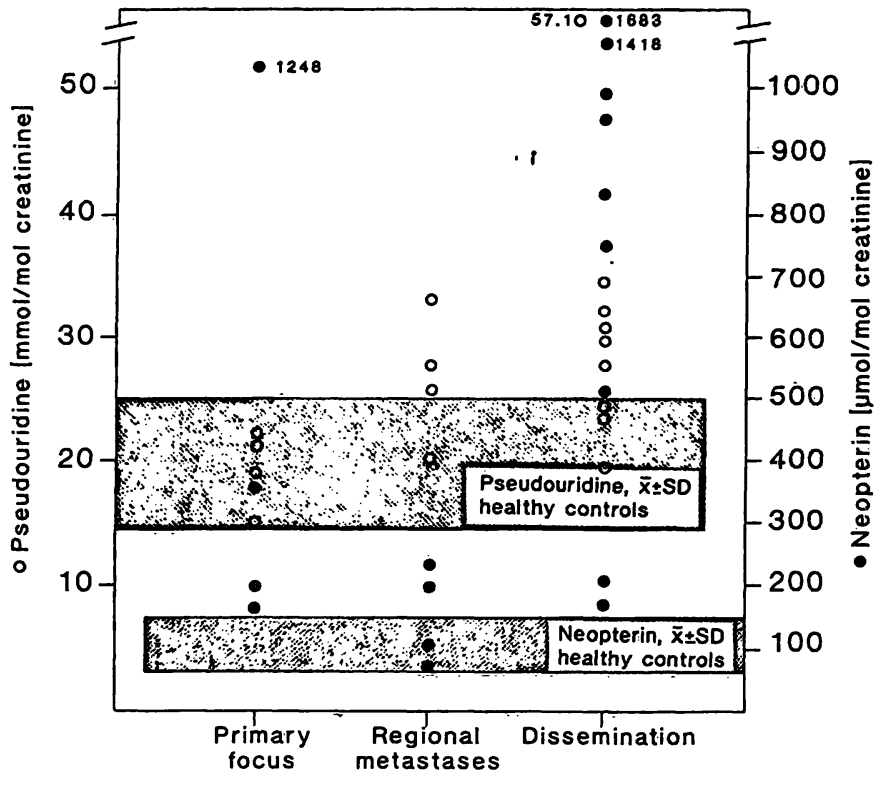

Fig. 3. The relationship between the stage of disease and urinary neopterin (closed circles) and pseudouridine (open circles) concentration in patients with adenocarcinoma of the large intestine.

could mean that not only malignant growth but also other influences e.g. degree of cell differentiation, are important in the induction of immune activation in patients.

In conclusion, urinary neopterin is a more sensitive biochemical marker of malignancy than pseudouridine. Simultaneous determination of neopterin, pseudouridine, and creatinine by HPLC is a simple, rapid, precise, and relatively inexpensive method, which can be useful in many medical laboratories.

\section{Acknowledgements}

The authors wish to thank Dr. Roman Dorociak for permission to study his patients, and Mrs. Teresa Hass for skilful technical assistance.

\section{References}

1. Huber, C., Batchelor, J. R., Fuchs, D., Hausen, A., Lang, A., Niederwieser, D., Reibnegger, G., Swetly, P., Tropmair, J. \& Wachter, H. (1984) Immune response-associated production of neopterin - release from macrophages primarily under control of interferon gamma. J. Exp. Med. 160, 310 316.

2. Werner, E. R., Bichler, A., Daxenbichler, G., Fuchs, D., Fulth, L. C., Hausen, A., Hetzel, H., Reibnegger, G. \& Wachter, H. (1987) Determination of neopterin in serum and urine. Clin. Chem. 33, 62-66.

3. Hausen, A., Fuchs, D., Konig, K. \& Wachter, H. (1982) Determination of neopterin in human urine by reversedphase high-performance liquid chromatography. J. Chromatogr. $227,61-70$.

4. Auzeby, A., Bogdan, A., Krosi, Z. \& Touitou, Y. (1989) Large-amplitude circadian variations of urinary neopterin in healthy man. Pteridines $1,17-18$.

5. Hausen, A., Fuchs, D., Reibnegger, G., Werner, E. R. \& Wachter, H. (1989) Neopterin in clinical use. Pteridines $l$, $3-10$.

6. Sander, G., Topp, H., Heller-Schöch, G., Wieland, J. \& Schöch, G. (1986) Ribonucleic acid turnover in man: RNA catabolites in urine as measure for the metabolism of each of three major species of RNA. Clin. Sci. 71, 367-374.

7. Weissman, S., Eisen, A. Z., Lewis, M. \& Karon, M. (1962) Pseudouridine metabolism. III. Studies with isotopically labelled pseudouridine. J. Lab. Clin. Med. 60, 40-47. 
8. Salvatore, F., Colonna, A., Costanzo, F., Russo, T., Esposito, F. \& Cimino, F. (1983) Modified nucleosides in body fluids of tumor-bearing patients. Recent Results Cancer Res. 84, 360-377.

9. Sorensen, S. H., Brown, D. A., Cooper, E. H., Kelly, K. A. \& Mac Lennan, I. C. M. (1985) Urinary pseudouridine excretion in myelomatosis. Br. J. Cancer 52, 863-866.

10. Borek, E., Sharma, O. K. \& Waalkes, T. P. (1983) New applications of urinary nucleoside markers. Recent Results Cancer Res. 84, $301-316$.

11. Heldman, D. A., Grever, M. R. \& Trewyn, R. W. (1983) Differential excretion of modified nucleosides in adult acute leukemia. Blood 61, 291-295.

12. Dieckhues, B. (1986) Identification of pathologic metabolic metabolites of melanin and melatonin metabolism in urine of patients with malignant melanoma of the choroid by means of high-pressure liquid chromatography (HPLC). Klin. Mbl. Augenheilk. 189, 34-35.
13. Tamura, S., Amuro, Y., Nakano, T., Fuji, J., Moriwaki, Y., Yamamoto, T., Hada, T. \& Higashino, K. (1986) Urinary excretion of pseudouridine in patients with hepatocellular carcinoma. Cancer 57, 1571 - 1575.

14. Graf, N., Bach, K., Frisch, B., Haas, H. J. \& Sitzmann, F. C. (1989) The clinical value of urinary excretion of pseudouridine in infancy and childhood. Klin. Pediatr. 201, 154-162.

15. Rasmuson, T., Björk, G. R., Hietala, S-O., Stenling, R. \& Ljunberg, B. (1991) Excretion of pseudouridine as an independent prognostic factor in renal carcinoma. Acta Oncologica $30,11-15$.

Prof. Dr T. Motyl

Department of Animal Physiology

Veterinary Faculty

Warsaw Agricultural University

Nowoursynowska 166

02-766 Warsaw

Poland 
